\title{
GESTIÓN ORGANIZACIONAL EN ENTORNOS COMPLEJOS POR PARTE DE LAS MIPYMES DEL SECTOR SERVICIOS DE LA CIUDAD DE SINCELEJO
}

\author{
ORGANIZATIONAL MANAGEMENT IN COMPLEX ENVIRONMENTS BY THE SERVICES \\ SECTOR MSMES CITY OF SINCELEJO
}

\section{GESTÃO ORGANIZACIONAL EM AMBIENTES COMPLEXOS PELO SETOR SERVIÇOS DAS PME SINCELEJO}

\author{
Por: DEL RIO VÁSQUEZ _ Jorge Luis
}

\begin{abstract}
Especialista en Investigación aplicada y Administración de la informática educativa, Universidad de Santander y Corporación Universitaria del Caribe, CECAR. Docente rol investigador Escuela de Ciencias Administrativas, Corporación Unificada Nacional de Educación Superior CUN. Email: jorge_ delrio@cun.edu.co,Colombia.
\end{abstract}

DOI: http://dx.doi.org/10.22267/rtend.171802.75

\section{RESUMEN}

Con el propósito de determinar los modelos de gestión organizacional en entornos complejos que practican las MIPYMES del sector servicios en la ciudad de Sincelejo - Sucre - Colombia, se adelanta la presente investigación. Para ello es importante tener en cuenta su gestión actual, toda vez que estas representan en su mayoría el tejido empresarial de las economías locales y nacionales.

El presente artículo pretende un análisis, a la luz de la teoría y antecedentes empíricos, que demuestre la relación entre la gestión organizacional y los índices de perdurabilidad o tasas de mortalidad de las MIPYMES del sector servicios, definiendo los principales enfoques de modelos de gestión organizacional en cabeza de autores como Koontz y Weihrich, (1998), De la Rosa, (2002), Wong, (2000), entre otros.

Finalmente, las MIPYMES del sector, desde su enfoque funcional u operativo e informalidad no se aprestan a un enfoque basado en procesos, por lo tanto, los esfuerzos en materia de fortalecimiento y consolidación empresarial, estarían encaminados al desarrollo de la gestión del talento humano, la gestión comercial, 
la gestión gerencial, la gestión de producción, la gestión financiera, la gestión tecnológica y la gestión de calidad.

Palabras clave: proceso, gestión, organización, competitividad.

JEL: M1.

\begin{abstract}
In order to determine standard organizational management in complex environments that practice MSMEs in the services sector in the city of SincelejoSucre-Colombia, this investigation is being conducted. It is therefore important to consider your current management, since these represent mainly the business of local and national economies.
\end{abstract}

The present article pretends an analysis, to the light theory and empirical antecedents, that show the relation between the management organizacional and the indexes of perdurabilidad or taxes of mortality of the MIPYMES of the sector service, defining the main approach of models of management organizacional in head of authors eat Koontz And Weihrich, (1998), Of Rosa, (2002), Wong, (2000), among others.

Finally, the MIPYMES of the sector, from his functional or operative approach and informality no aprestan even to approach based in processes, therefore, the efforts in matter of strengthening and business consolidation, would be directed to the development 0 f the management of the human talent, the commercial management, the managerial management, the management of production, the financial management, the technological management and the management of quality.

Key words: process, management, organization, competitiveness.

JEL: M1.

\title{
RESUMO
}

A fim de determinar a gestão organizacional padrão em ambientes complexos que praticam MPMEs no sector dos serviços na cidade de Sincelejo-Sucre-Colômbia, esta investigação está sendo conduzida. Por isso, é importante considerar a sua gestão corrente, uma vez que estes representam, principalmente, o negócio das economias locais e nacionais.

Este artigo tenta uma análise, à luz teoria e fundo empírica, mostrando a relação entre gestão organizacional e as taxas de setor de serviços MPMEs longevidade ou taxas de mortalidade que definem o foco principal dos modelos de cabeça gestão organizacional autores como Koontz e Weihrich, (1998), de la Rosa, (2002), Wong (2000), entre outros.

Finalmente, o sector MPME, desde a sua abordagem funcional ou operacional e informalidade não estão se preparando até mesmo abordagem de processo, portanto, os esforços no fortalecimento e consolidação, que tem por objectivo 
desenvolver a gestão de talentos humanos, gestão empresarial, gestão empresarial, gestão da produção, gestão financeira, gestão de tecnologia e gestão.

Palavras-chave: processo, gestão, organização, competitividade.

JEL: M1.

\section{INTRODUCCIÓN}

Actualmente las organizaciones deben hacerle frente a un entorno complejo y altamente competitivo, donde la globalización de la economía y el auge de mercados comunes generan una constante línea por conquistar clientes o usuarios, creándose un ambiente de competencia dinámico y exigente. Esto, se suma a que la era industrial ha sido reemplazada por la sociedad de la información y del conocimiento, generando la puesta en práctica de modelos de gestión organizacional más eficientes, en los cuales los servicios y la calidad se hacen esenciales. En este mismo orden, destacados expertos opinan que alrededor del $80 \%$ del valor de mercado de una organización reside en sus elementos intangibles (Bueno, 1998), lo cual hace de la gestión la principal tarea administrativa.

El sector de las MIPYMES dedicadas a la prestación de servicios en la ciudad de Sincelejo, está inmerso en esta dinámica de lo complejo, su promesa de servicio al mercado recae sobre un entorno altamente competitivo, donde la constante es el cambio de las condiciones micro y macroambientales. Desde esta perspectiva, las Pymes colombianas deben alinearse con las tendencias que impone la gerencia del siglo XXI, en donde la competitividad se convierte en el eje central del negocio y la producción de conocimiento (Villegas y Toro, 2009).

En este sentido, Brune y Belzunegui (2000) se pronuncian, destacando como bajo el nuevo entorno global y complejo de mayor competencia, la empresa clásica y grande se enfrenta a una economía de red y a la necesidad de contar con "una elevada flexibilización tanto cuantitativa como cualitativa, una disminución de plazos de fabricación o atención, unos adecuados niveles de calidad y fiabilidad de los productos o servicios, etc., y en consecuencia, un gradual incremento de la automatización y estandarización.

Por lo tanto, en los últimos años el aumento de la tasa de mortalidad de las MIPYMES del sector es exponencial, al primer año de creadas, sobreviven 55 empresas de cada 100. La informalidad de las mismas (cabe destacar que el 97.2\% de los establecimientos reportados en Sucre correspondió a empresas de hasta 10 trabajadores), (Pérez, 2011), y la afectación estructural a la economía local y regional, son la causa y consecuencia más evidente que asoma este panorama.

Cabe destacar que, la falta de perdurabilidad empresarial, en el departamento de Sucre y su capital Sincelejo, se evidencia, en la constitución de sus 922 empresas, que representan el $67.2 \%$ del total del municipio, las cuales fueron constituidas en los últimos cinco años, lo que permite decir que el parque empresarial de Sincelejo es relativamente nuevo (Cámara de Comercio de Sincelejo, 2013) y la mayoría son de origen familiar. 
Estas MIPYMES dedicadas en mayor medida al comercio, los servicios de salud, estéticas, hospedaje y restaurante, son el resultado de emprendimientos espontáneos por parte de particulares, quienes con niveles básicos de formación académica incursionan en el mercado donde aprenden el oficio desde la gestión informal del negocio y sus clientes, formulando planes estratégicos frente al entorno complejo que les toca enfrentar. En efecto, las empresas de menor tamaño (micro, pequeñas y medianas empresas) y en especial las nuevas, por su flexibilidad y potencialidad en términos de costos labores y empleo, tienen efectos importantes en los países en desarrollo, dinamizan economías emergentes, aún más, considerando que son el origen de donde surge la clase media.

En consecuencia, hablar de las MIPYMES en economías intermedias y locales, es hablar de la dinámica que soporta a las mismas, su impacto en la generación de bienes y servicios y la creación de empleos sostenibles, por lo tanto, su estudio y el análisis de su impacto y naturaleza debe partir de su gestión y con ello sus desenvolvimiento en entornos turbulentos o complejos.

De esta manera, gestionar organizaciones en entornos complejos supone la admisión y manejo de variables micro y macroambientales que en marcan a las empresas dentro de dinámicas de competitividad, demandando modelos estratégicos de conducción de recursos y personal.

Las teorías del caos y de la complejidad están suponiendo la aparición de nuevas perspectivas en el entendimiento del fenómeno organizativo. En este sentido, y al igual que en la década de los 60 el concepto de sistema abierto supusiese un cambio de paradigma en la teoría organizativa, en este traspaso de siglo también hay una serie de conceptos vinculados a las teorías del caos y de la complejidad que están llamando a un nuevo cambio de paradigma o, en el peor de los casos, a la inclusión de una forma más de concebir las organizaciones, de una nueva metáfora (Morgan, 1986).

Por otra parte, este entorno es complejo, entendiendo por complejo, en palabras de Morín (2007), la complejidad es, efectivamente, el tejido de eventos, acciones, interacciones, retroacciones, determinaciones, azares, que constituyen nuestro mundo fenoménico, apreciable solo en su dinámica y adaptación al mismo. Lo anterior abre paso al entendimiento de las empresas actuales como sistemas complejos, al igual que lo es el medio ambiente o entorno en el que se desenvuelven.

Desde las teorías del caos y de la complejidad comienzan a emerger modelos más antirracionalistas de la comprensión del fenómeno organizativo que dejan de lado los conceptos anteriores de equilibrio y estabilidad proponiendo otros nuevos. Frente al pensar prototipo del modelo racional de organización (Peiró, 1993 y Munduate, 1997) según el cual debemos analizar el entorno, diseñar nuestras organizaciones en referencia a sus objetivos racionales y conscientes y también de acuerdo con dicho entorno y, finalmente, buscar un ajuste organización - entorno, las teorías del caos y de la complejidad proponen la emergencia de un orden, de una organización, que acontece de manera espontánea una vez que se dan unas condiciones determinadas (condiciones de alejamiento del equilibrio, condiciones que sitúan al sistema en el borde del caos, etcétera). 
Levy (1994), reconoce cinco elementos mediante los cuales la Teoría del Caos realiza aportes a la conducción de las organizaciones:

1) La planificación a largo plazo es muy difícil: con el paso del tiempo las pequeñas perturbaciones pueden multiplicarse debido a las relaciones no lineales y a la naturaleza no estable del sistema. La utilización de modelos más complejos y precisos no siempre es garantía de la obtención de mejores pronósticos.

2) Las organizaciones no alcanzan un equilibrio estable: los sistemas ampliamente conectados con el entorno no alcanzan un equilibrio estable, no logrando pasar exactamente por el mismo lugar más de una vez.

3) Un cambio dramático puede producirse en forma inesperada: la aparición de cualquiera de las "cuatro fuerzas competitivas de Porter" puede generar una repercusión muy importante sobre la organización.

4) Pueden hacerse pronósticos a corto plazo y predicciones de patrones: en los sistemas caóticos se observa, en el corto plazo, un grado sorprendente de orden. 0 sea que las predicciones sobre las que se basa la selección de la estrategia, pueden tener un aceptable grado de cumplimiento en horizontes temporales cortos.

5) Se necesitan pautas para enfrentar la complejidad y la incertidumbre: es necesario adoptar estrategias simplificadoras para orientar las decisiones que se toman en un mundo de muy complejas interacciones.

La existencia de dichos entornos complejos, es lo que permite hablar de modelos de gestión, que bien se definen para la administración racional, como aquellas estructuras que suministran un medio para analizar y sintetizar situaciones y sistemas complejos, los modelos son el punto de partida para la administración racional, y suministran un medio para analizar y sintetizar situaciones y sistemas complejos. Ahora bien, los modelos de gestión se definen como la administración racional y estratégica de las organizaciones, optimizando los recursos para analizar y sintetizar situaciones y sistemas complejos. La importancia de los modelos se enfoca específicamente en que son una herramienta que contribuye en convertir una verdad absoluta en una verdad relativa, convirtiéndose de esta manera en un apoyo diagramático de todas aquellas respuestas que la ciencia trata de dar.

En tal sentido se conciben diferentes modelos de gestión organizacional clasificados de acuerdo al énfasis que estos hacen sobre los recursos y el talento con que cuenta la organización en un entorno complejo, así se tiene El Modelo Administrativo (Koontz y Weihrich, 1998), son aquellos que abordan el fenómeno organizacional con una filosofía global que permita hacer uso de herramientas y técnicas como medios utilizables en determinados momentos y no con fines en sí mismas; Según esta perspectiva, los roles o papeles son fundamentales especialmente en la alta gerencia en función de la conducción general de la empresa, situación que se ve aumentada en la medida en que la unidad de producción se hace más compleja y se trata de adaptar a escenarios de creciente competitividad. 
Por su parte, el Modelo de la Dinámica Organizacional (De la Rosa, 2002), considera los insumos como factores que ingresan a las organizaciones provenientes del entorno externo. Los insumos incluyen: el capital, la tecnología, la materia prima y el recurso humano, los productos son los indicadores de la efectividad de la organización; el planteamiento adoptado resalta los componentes fundamentales que integran una organización en un ambiente dinámico. Considerando a los individuos, a los grupos laborales, a la tecnología, el diseño de la organización, procesos organizacionales y al ambiente externo.

Así mismo, el Modelo de los Procesos Motivacionales Básicos (Wong, 2000), Para comprender la motivación humana es necesario examinar las fuerzas internas de los individuos y sus entornos que le suministran retro-información y refuerzan su intensidad y dirección con el trabajo. Enfatiza la naturaleza cíclica de motivación y muestra que los individuos se encuentran en constante estado de desequilibrio, pugnado continuamente para satisfacer una amplia variedad de necesidades.

De igual manera, está el Modelo Situacional Sobre el Liderazgo (Blanchard, 1999), adiciona la dimensión de la madurez de los seguidores a la situación del liderazgo. La aceptación que dan al termino es que la madurez no tiene nada que ver en la edad cronológica; por el contrario, la madurez de individuos o grupos se refiere a su capacidad de asumir la responsabilidad de dirigir la propia conducta. En el liderazgo situacional, la madurez se define como la capacidad y dimensión de los individuos a asumir la responsabilidad de dirigir su propia conducta. Estas variables de la madurez deberían considerarse exclusivamente en relación con determinada tarea que debe efectuarse.

Por la misma vía, está el Modelo para la Toma de Decisiones (Hellriegel, Jackson y Slocum, 2005), en este modelo, el proceso comienza cuestionando la retro-alimentación negativa a una oportunidad, en esta faceta, la organización hace hincapié en las relaciones de contexto con el medio externo, tanto en el aprovisionamiento de insumos, recursos humanos, clientes, y en lo externo en cuanto a la medición cuantitativa y proyección de sus resultados. La toma de decisiones tanto individual como de grupo debe estar plenamente reforzada por mecanismos de control, evaluación o monitoreo, con el fin de identificar y establecer mecanismos correctivos en el proceso de gestión.

Finalmente está el Modelo del Proceso de la Comunicación (Jablin, 1986), es aquel modelo que muestra que el emisor parte de un significado, puede trazarse una distinción útil entre dos clases de comunicación: la eficaz y la buena. La comunicación eficaz se da cuando el emisor logra del receptor los resultados deseados. La buena comunicación se efectúa cuando la compresión del receptor coincide con el significado que el emisor desea transmitirle.

Por su lado, las MIPYMES, obedecen a una clasificación no exenta de debate y polémica, toda vez que los criterios nacionales e internacionales para la clasificación de las organizaciones empresariales, se ha trasformado conforme las dinámicas del mercado y los giros económicos. Sin embargo, la definición vigente es la acuñada por Ley 590 de 2000, por la cual se dictan disposiciones para promover el desarrollo de las micro, pequeñas y 
medianas empresas, donde en su artículo 2 se les define de la siguiente manera, para todos los efectos, se entiende por micro, pequeña y mediana empresa, toda unidad de explotación económica, realizada por persona natural o jurídica, en actividades empresariales, agropecuarias, industriales, comerciales o de servicios, rural o urbana, que responda a los siguientes parámetros:

1. Mediana Empresa:

a) Planta de personal entre cincuenta y uno (51) y doscientos (200) trabajadores;

b) Activos totales por valor entre cinco mil uno (5.001) y quince mil (15.000) salarios mínimos mensuales legales vigentes.

2. Pequeña Empresa:

a) Planta de personal entre once (11) y cincuenta (50) trabajadores;

b) Activos totales por valor entre quinientos uno (501) y menos de cinco mil (5.001) salarios mínimos mensuales legales vigentes.

\section{Microempresa:}

a) Planta de personal no superior a los diez (10) trabajadores;

b) Activos totales por valor inferior a quinientos uno (501) salarios mínimos mensuales legales vigentes.

PARAGRAFO 1o. Para la clasificación de aquellas micro, pequeñas y medianas empresas que presenten combinaciones de parámetros de planta de personal y activos totales diferentes a los indicados, el factor determinante para dicho efecto, será el de activos totales (Diario oficial, 2000).

\section{MÉTODO}

El presente artículo se adelantó con los siguientes parámetros metodológicos: para la ubicación de los documentos bibliográficos se utilizaron varias fuentes documentales, primarias y secundarias. Se realizó una búsqueda bibliográfica E-libro: Colección de títulos en español, monografías, revistas y libros, utilizando los descriptores: gestión por procesos, modelos de gestión, entornos complejos, competitividad, MIPYMES.

Los registros obtenidos oscilaron entre 45 y 56 registros tras la combinación de las diferentes palabras clave. También se realizó una búsqueda en internet en el buscador "google académico" con los mismos términos. Se seleccionaron aquellos documentos que informasen sobre los aspectos formales que debía contener una revisión, la lectura crítica de documentos, las etapas de realización de una revisión bibliográfica o la elaboración de mapas mentales o mapas conceptuales. 


\section{DESARROLLO DEL TEMA}

\section{La gestión organizacional en entornos complejos}

La definición más aceptada del concepto gestión se relaciona directamente con el de administración, y significa "hacer diligencias conducentes al logro de unos objetivos" (Ramírez, 2005, p. 14), estas diligencias implican recursos y personas que sistemáticamente direccionan sus esfuerzos hacia un mismo rumbo. En palabras de Fajardo, "conjunto de conocimientos modernos y sistematizados en relación con los procesos de diagnóstico, diseño, planeación, ejecución y control de las acciones teológicas de las organizaciones en interacción con un contexto social orientado por la racionalidad social y técnica" (Fajardo, 2005. Pág. 85).

De acuerdo a la aceptación del concepto de gestión, las MIPYMES del sector servicio, han hecho frente en los últimos años a un entorno cambiante y competitivo, que ha mostrado que una constante es el cambio y la gestión su nuevo paradigma. Este cambio ha exigido de las mismas verdaderas estrategias de adaptación y flexibilidad en todo el mundo, como bien lo indica el autor, siguiendo a Camisón (2000), el nuevo paradigma empresarial reclama industrias de menor tamaño que respondan con rapidez a los requerimientos del mercado. Esas estructuras organizativas tienen tres características fundamentales: la flexibilidad, la competitividad y la adaptación al cambio. En este sentido, las micro, pequeñas y medianas empresas son una referencia en cuanto a capacidad de anticipación y respuesta a los retos del entorno (Camisón, 1997).

Sin embargo, estas MIPYMES prestadoras de servicios en la ciudad de Sincelejo, no siempre tienen la capacidad de adaptación y desarrollo necesarios para sobrevivir al entorno local y nacional, muchas de estas, son arrasadas por las grandes superficies de servicios, los centros comerciales y siempre, la gestión organizacional, basada en experiencias subjetivas por parte de sus propietarios y/o gerentes, que para el caso resultan ser la misma persona.

El hecho de tener que hacer frente a los entornos cada vez más cambiantes y turbulentos a los que se enfrentan estas organizaciones, lleva a la necesidad de que toda MIPYME se plantee como objetivo fundamental, la mejora continua y permanente de su competitividad (Aragón y Sánchez, 2003), esto implica gestionar procesos estratégicos, misionales y de apoyo para no solo adaptarse sino ser competitivas.

Ahora bien, la medición de este concepto de gestión puede hacerse en las investigaciones desde diversos ámbitos. En efecto, puede ser efectuada a través de indicadores financieros y de mercado; por la presencia de competencias propias del empresario y de los gerentes; a través de las estrategias competitivas implantadas por la organización; así como por factores del entorno, como barreras de entrada y salida, competidores y proveedores, esto es, se puede vislumbrar desde lo que se considera en conjunto, modelos de gestión como enfoque estratégico y práctico. 
Por lo tanto, las MIPYMES del sector servicios de la ciudad, deben ante todo, organizar su estrategia de permanencia y crecimiento frente al entorno, basadas en la gestión de los factores organizacionales, como lo indica Camisón (2000), entre los factores que determinan el éxito de las pequeñas y medianas empresas, se encuentran los siguientes: recursos financieros, recursos humanos, recursos organizativos, recursos comerciales y recursos tecnológicos, y son estos mismos los factores a gestionar desde un modelo de gestión que responda a las particularidades de una empresa y su entorno.

De esta manera, queda evidenciado, que para las MIPYMES del sector servicios, una nueva visión de lo organizacional, basada en la gestión de procesos claves, se hace necesaria y pertinente, lo cual conduce a los propietarios a una cualificación efectiva a cuentas de una reestructuración de sus tareas hacia la conformación de modelos formales de gestión para hacerle frente al entorno.

\section{Enfoque funcional vs. Enfoque por procesos en las MIPYMES del sector servicios}

Cuando se indaga en las MIPYMES de la ciudad, nos encontramos de forma inmediata con estructuras organizacionales informales y enfocadas desde lo funcional, empresas divididas bajo esquemas lineales de departamentalización que, no tienen mapas de procesos, al menos básico, como se pudo constatar en una prueba piloto reciente a 11 MIPYMES del sector servicio. Esto llama poderosamente la atención si se tiene en cuenta que, en la administración actual la gestión por procesos constituye un reto para todas las organizaciones dada las circunstancias actuales del mercado donde la competitividad rige en el éxito o fracaso de los negocios (Marrero, Domínguez y Fajardo, 2012).

Ahora bien, las MIPYMES del sector servicio, en un entorno complejo requieren con suma urgencia cambiar de enfoque, en tal sentido, el enfoque basado en procesos parte de la idea de que "las empresas son tan eficientes como lo son sus procesos" (Amozarrain, 1999), reconoce que todo trabajo dentro de la organización se realiza con el propósito de conseguir algún objetivo, y que el objetivo se logra más eficazmente cuando los recursos y las actividades relacionadas se gestionan como un proceso. Esto exige a las organizaciones en mención, la reelaboración de sus procesos, para una gestión más eficiente y eficaz del servicio.

Este nuevo enfoque requiere de la conformación de un mapa de procesos y una gestión basada en los mismos para las MIPYMES, toda vez que, de acuerdo a Edgar José Anaya Vélez, es evidente la falta de planeación estratégica, las acciones están enfocadas en el servicio al cliente y disminución de costos, sin tener una visión global de la organización para su gestión. La gestión de los directivos reconoce la importancia de la forma de gobierno y la operación eficiente, sin muestras significativas de liderazgo y creatividad, por lo que sin duda, se caracterizan por una administración de tipo operativo (Anaya, 2014).

Por lo tanto, el inicio de cualquier programa de consolidación de las MIPYMES del sector servicios, con fines de aumentar sus tasas de perdurabilidad en el mercado y su competitividad, debe estar sobre la base de la gestión por procesos, superando el obsoleto 
enfoque funcional u operativo, que se limita a la administración de costos, con bajos índices de eficiencia y eficacia.

\section{Aproximación empírica a los modelos de gestión en las MIPYMES sector servicios de la ciudad de Sincelejo}

Un modelo demanda recursos, personas, tiempos y relaciones sistemáticas entre las partes, con una finalidad esperada. En tal sentido, suministra medios para analizar, sintetizar y afrontar situaciones y sistemas complejos (Mascareñas, 2001). Es así como los modelos de gestión ofrecen formas de actuar sobre las personas, ya que éstas le confieren vitalidad a las actividades y procesos que en la organización se ejecutan, por lo tanto, los modelos son fundamentales para el funcionamiento de las empresas, enfrentadas a escenarios cada vez más turbulentos (Alvesson, 1977; Becker y Gerhartm 1996; citado por Davel y Vergara, 2001).

Ahora bien, lo opuesto a los modelos de gestión organizacional es la informalidad, la praxis operativa que se conjuga en el día a día, la departamentalización lineal carente de gestión y liderazgo, que encuentra a la MIPYME sin capacidad de respuesta frente al mercado y con pocas expectativas de durabilidad en el entorno complejo que le toca enfrentar. Son numerosos los resultados arrojados al respecto y asociados a la mortalidad de las MIPYMES en Colombia, lo que nos coloca frente a una relación causa efecto, entre la gestión de estas y su competitividad o supervivencia en el mercado.

Uno de estos estudios destaca la importancia que tiene la planeación estratégica como factor determinante para la longevidad de las empresas (Panjwani et al., 2008). Según los resultados es preocupante que solo el $8.57 \%$ de las empresas del estudio consideren que estas acciones hayan incidido en su perdurabilidad. Así mismo, la gestión del conocimiento en la organización es un factor determinante de éxito, según Nonaka (1991) en una economía donde lo único cierto es la inestabilidad, el conocimiento es una fuente segura de ventaja competitiva sostenible, por lo anterior, es desconcertante que según los resultados, solo el $28.57 \%$ consideren que estas acciones han incidido en la perdurabilidad.

Este panorama no inhibe el asunto de los costos de operación, las MIPYMES con frecuencia incurren en sobre costos en sus procesos, como lo destaca la gestión de la eficiencia y la eficacia en los procesos representan para la empresa comercial reducciones significativas en los costos de operación y comercialización y por ende, mayor utilidad operativa, lo que se traduce en mayor rentabilidad para los propietarios maximizando su riqueza.

Por lo tanto, la administración con un enfoque funcional u operativo de las MIPYMES del sector servicio en la ciudad de Sincelejo, tiene una relación directa con las altas tasas de mortalidad y estancamiento organizacional que padecen, según la falta de compromiso con el desarrollo social, la responsabilidad social y la cohesión social para la acción, lo que debilita a la organización. Las empresas en general demuestran poco interés en la gestión de protocolos de relevo generacional, lo que es, un factor determinante en la longevidad 
de las empresas; presentan una ineficiente gestión del conocimiento y débiles políticas de vinculación y retención de los empleados, a esto se sumó su poca gestión tecnológica y su casi nulo conocimiento acerca de la calidad (Anaya, 2014).

\section{CONCLUSIONES}

Luego de la anterior revisión documental acerca de los modelos de gestión organizacional y su injerencia en las MIPYMES del sector servicios de la ciudad de Sincelejo, como factor de perdurabilidad y desarrollo en entornos complejos, se tienen las siguientes conclusiones:

- Los entornos complejos representan el carácter de los mercados actuales, plagados de cambios impredecibles, alta competencia e innovación permanente, frente a lo cual las micro, pequeñas y medianas empresas deben hacer frente desde sus recursos y formas de administración.

- MIPYMES del sector servicios de la ciudad de Sincelejo, deben superar en su gran mayoría, el enfoque de administración funcional u operativo, pasando a un enfoque de gestión por procesos, donde, a partir de un liderazgo efectivo, se asuma a la organización como un sistema abierto que se adapta estratégicamente al entorno para evolucionar.

- Existe una relación causa efecto entre los enfoques de administración de las MIPYMES y su perdurabilidad en el mercado, toda vez que los entornos complejos requieren de una gestión eficiente y eficaz de factores claves de éxito que las empresas desconocen desde su práctica.

- Los altos niveles de informalidad de las MIPYMES del sector, es una de las principales razones por las cuales no se consolidan modelos de gestión por procesos que integren lo gerencial, lo comercial, lo productivo, lo financiero, lo tecnológico, lo humano y la mejora continua.

- Los modelos de gestión organizacional implementados en las o MIPYMES del sector servicios de la ciudad de Sincelejo, apuntarían al desarrollo del talento humano y a la gestión tecnológica, puntos críticos que afectan los costos de operación de las mismas, que, de acuerdo a investigaciones adelantas por diversos autores, son relativamente altos. 


\section{REFERENCIAS}

(1) ANAYA, Edgar. (2014). Factores determinantes de la perdurabilidad de las empresas MIPYMES comerciales en el municipio de Sincelejo - Sucre: apreciaciones de los directivos. Universidad Nacional de Colombia Facultad de Ciencias Económicas. Sincelejo, Colombia. Página. 29.

(2) AMOZARRAIN, Manuel. (1999). La gestión por procesos. Editorial Mondragón Corporación Cooperativa, España. Pág. 2.

(3) ARAGÓN, Antonio.; SÁNCHEZ, Gregorio. (2003). Orientación estratégica, características de gestión y resultados: un estudio en las pyme españolas. ICE Tribuna de Economía, 809, pp. 169187.

(4) BLANCHARD, Ken. (1999). Leadership and the One Minute Manager, William Morrow. New y Updated. U.S.A. Pág. 39.

(5) BUENO, Eduardo. (1998). El capital intangible como clave estratégica en la competencia actual. Boletín de Estudios Económicos. 1998. Vol. 53(164). Madrid, España.

(6) CÁMARA DE COMERCIO DE SINCELEJO. (2013). Base de Datos 2013. Sincelejo.

(7) BRUNET Ignasi; BELZUNEGUI Angel (2000). Empresa y estrategia en la perspectiva de la competencia global. Ariel Economía. Barcelona, España. Pág. 256.

(8) CAMISÓN, Cesar. (1997). La competitividad de la pyme industrial española. Estrategias y competencias distintivas. Biblioteca Civitas Economía y Empresa. Colección Empresa: Madrid, España. Párrafo 14.

(9) CAMISÓN, Cesar. (2000). Reflexiones sobre la investigación científica de la pyme. Investigaciones Europeas de Dirección y Economía de la Empresa, 6 (2), pp. 13-30. México D.C. México.

(10) CAMISÓN, Cesar. (2000). Reflexiones sobre la investigación científica de la pyme. Investigaciones Europeas de Dirección y Economía de la Empresa, 6 (2), pp. 13-30. México D.C. México.

(11) DE LA ROSA ALBURQUERQUE, Ayuzabet. (Julio 2002). Teoría de la Organización y Nuevo Institucionalismo en el Análisis Organizacional. Administración y Organizaciones. No. 8; Año 4, 13-44. México D.C. México.

(12) DIARIO OFICIAL No. 44.078 de 2000.

(13) DAVEL, E. y VERGARA, S. (2001). Gestión de personas y subjetividades. Sao Paulo: Atlas. Núm. 493-496.

(14) PANJWANI, A., AGgARWAL, V.; DHAMEJA, N. (2008). Family Business: Yesterday, Today, Tomorrow. Indian Journal of Industrial Relations, 44(2). 
(15) HELLRIEGEL, D. JACKSON, S. y SLOCUM, J. (2005). Administración. Un enfoque basado en competencias. 10ma.edición. Thomson: México.

(16) KOONTZ Harold. WEIHRICH Heinz. (1998). Administración una perspectiva global. Mc GrawHill: México. Pág. 203.

(17) JABLIN, FRED, (1986). Estudio de la comunicación organizacional: su evolución y su futuro, la comunicación humana. La Comunicación Humana: Ciencia Social. McGraw Hill: México. Pág. 35.

(18) FAJARDO, Carlos. (2005). Gestión, Entorno y Competitividad de las Organizaciones. Citado por Vicerrectoría de investigación y transferencia. Universidad la Salle. Bogotá, Colombia. Pág. 85.

(19) LEVY David. (1994). Caos Teoría y Estrategia: Teoría, aplicaciones y de gestión Implicaciones Strategic Management Journal, 15, 176.

(20) Marrero, Martha. Domínguez, Julián. y Fajardo, Bleittys, (2012). La Gestión por Procesos como técnica para el éxito de las organizaciones. Artículo. Universidad de la Isla de la Juventud. Cuba. Disponible en: http://bvs.sld.cu/revistas/infd/n1512/infd03212.htm

(21) MORIN, Edgar. (2007). Introducción al pensamiento complejo. Gedisa: Barcelona.

(22) MASCAREÑAS, Juan. (2001). Manual de funciones y adquisiciones de empresas. Mc Graw Hill: España. Pág. 83.

(23) MUNDUATE, Lourdes. (1997). Conflicto y negociación. Madrid: Pirámide. Pág. 73.

(24) NONAKA, Ikujiro. (1991). The Knowledge Creating Company. Hardvard Business Review, November. /December, pp. 96-104.

(25) PÉREZ, Claudia Milena. (2011). Corporación observatorio del mercado de trabajo en Sucre. Sincelejo. Pág. 39.

(26) PEIRÓ, José. Manuel. (1983). Psicología de las organizaciones. Madrid: UNED. (5ª̣). Pág. 54.

(27) RAMÍREZ, Carlos. (2005). Fundamentos de Administración. Bogotá: Textos Universitarios. Pág. 202.

(28) VILLEGAS Dora, y TORO Ivan. (2009). Las pymes: una mirada a partir de la experiencia académica del mba. Universidad EAFIT. Medellín - Colombia. Articulo revista. Pág. 88.

(29) WONG, Roderick. (2000). Motivation. A Biobehavioural Approach. Cambridge: Cambridge University Press. Pág. 2 -3. 